\title{
Supplier side optimal bidding strategy for electricity market using bacterial foraging optimization algorithm
}

\author{
Uday Kiran $L^{1}$, Siva Nagaraju $\mathbf{S}^{2}$, Chandram Karri ${ }^{3}$ \\ ${ }^{1,2}$ Department of Electrical Engineering, JNT University, India \\ ${ }^{3}$ Department of Electrical Engineering, BITS-PILANI K.K Birla Goa Campus, India
}

\section{Article Info \\ Article history: \\ Received Feb 9, 2020 \\ Revised Apr 25, 2020 \\ Accepted May 12, 2020 \\ Keywords: \\ Bacterial foraging optimization algorithm Electricity power market Supplier side optimal bidding strategy}

\begin{abstract}
In this article, bacterial foraging optimization (BFO) algorithm is developed for single side optimal bidding strategy in an electricity market. Optimal bidding strategy is one of the important functions in the electricity market along with forecasting of the electricity price and the profit based unit commitment. The prime objective of generating company (Genco) is to maximize their profit when they participate in the bidding process. The BFO algorithm has been used to maximize the probability density function (pdf). In the second stage the BFO algorithm is again applied to maximize the profit of the suppliers. The proposed algorithm is developed in MATLAB (Version, 2019) and tested on standard test case available in the literature. Also, the simulation results are presented and compared. It is noticed that the proposed method yields the best results in terms of profit.
\end{abstract}

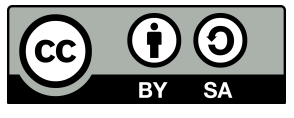

\section{Corresponding Author:}

Chandram Karri

Department of Electrical Engineering

BITS-PILANI K.K Birla GOA CAMPUS

NH 17B Bypass Road Zuarinagar Goa-403726, India

Email: chandramk2006@yahoo.co.in

\section{INTRODUCTION}

Electrical power is one of the most important infrastructure components for the economic growth and welfare of the developed countries. The demand for electrical power has increased rapidly due to several reasons [1]. In order to meet the increasing power demand, massive addition to the installed generating capacity is required. Due to the unique characteristics of the electricity, independent system operator (ISO) and regional transmission organization (RTO) are responsible to balance the power generation and demand [2]. In realtime power system operation, ISO forecasts and schedules generation to assure that the sufficient generation and back-up power is available to meet unexpected demand or generation loss. It must be a non-commercial organization, neutral and independent of commercial players. Currently, Nine ISO/RTO are responsible for the operation in the electricity markets.

In the deregulated power markets, the bids are submitted by the Genco's to the power exchange (PX) to buy and sell of the electric power. They gradually build their offers strategically to intensify their profits. This process is called bidding strategy [3]. The electricity market has been ruled by the bidding strategies. These strategies also grab the bidder's attention with the advantage of increasing profits. Flow of information in the electricity market is given in Figure 1 [4]. 


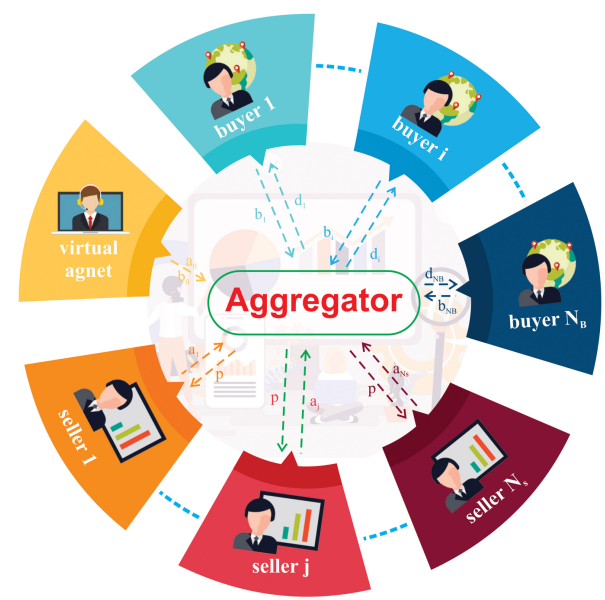

Figure 1. Flow of information in the electricity market

Several researchers in the past five decades used various mathematical techniques such as conventional, naturally inspired algorithms and hybrid methods for solving bidding strategy in the electricity markets. Highlights of these techniques are provided in [5]. The emerging electricity market has been analyzed by applying game theory in [6]. Network optimization technique is adopted for Nash equilibrium bidding strategy in [7]. The bidding strategy problem has been solved effectively using Monte Carlo simulation [8]. Discrete-state and discrete-time Markov decision process has been applied for optimal multi-period bidding strategy [9]. A Lagrangian relaxation [10] based approach has been adopted for strategic bidding. Strategic bidding problem for competitive power suppliers in the England - wale electricity markets is provided in [11, 12]. These algorithms such as genetic algorithm (GA) [13], particle swarm optimization algorithm (PSO) [14], differential evolution (DE) [15], invasive weed optimization (IWO) [16] and krill herd algorithm (KHA) [17], bacterial foraging algorithm [18], agent-based algorithm [19], bat inspired algorithm [20] have been applied in solving the DSOBS problem. Advantages and limitations of these methods are explained by few researchers. Some other algorithms [21, 22] by combining two or more algorithms have been developed to get global optimal solution for the DSOBS problem due to the complexity involved in the problem.

Bacterial foraging optimization (BFO) algorithm has been applied to solve various complex engineering problems. It is inspired by the social foraging behavior of Escherichia coli. Biology behind the foraging strategy of E. coli is used as an optimization algorithm. Scholars have been using the BFO algorithm with other available methods to enhance the local and global search properties. The BFO algorithm has been applied in solving several real time problems in engineering. Also, it has been found that the BFO algorithm is capable to provide the global solution. In contrast, mathematical modelling and modification of the algorithm is a major part of the research on BFO algorithm. Better solution can be achieved by the proper selection of the control parameters and updation of the algorithm. These observations motivated to introduce the BFO algorithm for solving single sided optimal bidding strategy in this article.

The contributions of the article are listed below : 1) Bacterial foraging optimization algorithm has been proposed for solving suplier side optimal bidding strategy. 2) The proposed algorithm has been coded in MATLAB (2019 version). 3) The BFO algorithm is tested on IEEE 30 bus system and results are provided. 4) The results like powers, profits and marginal cost price of the proposed method have been compared with the existing techniques.

The proposed bacterial foraging optimization algorithm is developed in MATLAB (Version 2019) and tested on standard test case available in the literature. The code has been executed on personal laptop (8 gb RAM, intel i5 processor, $2.3 \mathrm{GHz}$ ). The remaining paper is organized as follows: section 2 is bidding strategy problem formulation, section 3 is bacterial foraging optimization, section 4 is development of the algorithm, section 5 is case study, and section 6 is conclusion.

\section{PROBLEM FORMULATION}

In this section, a brief description about supplier side bidding strategy is provided. Also, mathematical formulation is given. The above are described in the following sections. 


\subsection{Bidding strategy}

In an open electricity market, the hourly aggregate supply is matched to hourly market clearing price (MCP) by the market operator. An optimal bidding strategy is mandatory for the Genco's in order to maximize its profits. In the deregulated power markets, Genco's build their offers to maximize profits. It is called strategic bidding.

\subsection{Mathematical formulation}

At the equilibrium point, The ISO matches the price for suppliers and consumers. At this point, the price is same for both the players. This price is called marginal cost price. Bidding curve of a suppliers is provided in Figure 2. The curve is mathematically modelled as follows:

$$
a_{i}+b_{i} P_{i}=M C P \quad i=1 \text { to } n
$$

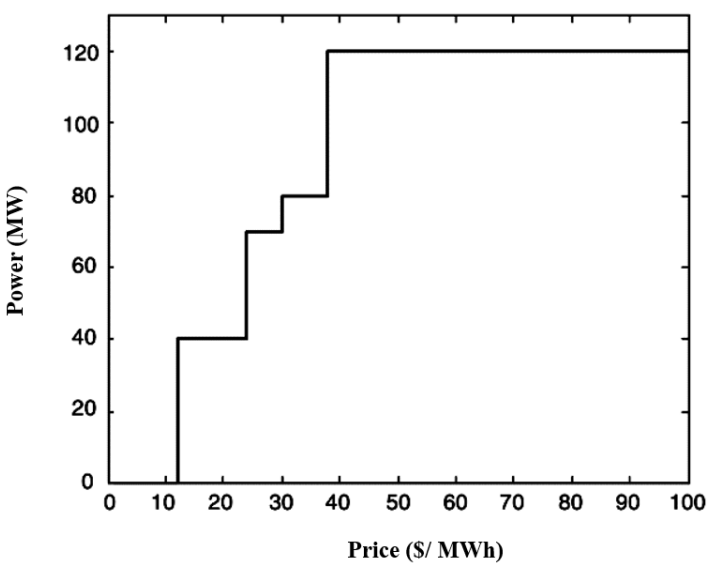

Figure 2. Bidding curve of a suppliers

\subsubsection{Objective function}

The profit of supplier and consumer is determined using the following equations.

$$
\text { Supplier }_{i} \text { profit }=M C P \times P_{i}-C_{i}\left(P_{i}\right)
$$

here, supplier s $_{i}$ ost function is

$$
C_{i}\left(P_{i}\right)=e_{i} P_{i}+f_{i} P_{i}^{2}
$$

In an ideal case, the power generation is equal to the power demand. In the pool market, when the price elasticity is present, the power demand varies. The mathematical equation of the cumulative fore-casted pool demand $(\mathrm{QMCP})$ is

$$
Q_{M C P}=Q_{O}-K \times M C P
$$

\subsubsection{Equality constraint}

The power equality constraint is

$$
\sum_{i=1}^{n} P_{i}=Q_{M C P}
$$

Expression of the marginal cost price (MCP) is derived from the above equations and provided below.

$$
M C P=\frac{Q_{0}+\sum_{i=1}^{n} \frac{a_{i}}{b_{i}}}{K+\sum_{i=1}^{n} \frac{1}{b_{i}}}
$$

The expressions for power and load are derived in terms of the MCP and bidding co-efficients and given blow.

$$
P_{i}=\frac{M C P-a_{i}}{b_{i}}
$$




\subsubsection{Inequality constraints}

Generator inequality constraint is

$$
P_{i}, \min \leq P_{i} \leq P_{i}, \max
$$

\subsection{Probability density function (pdf)}

Consumers and suppliers set the MCP to maximize their profits when they know their bidding coefficients. Each GENCO can guess their competitors bidding coefficients using the probability density function (pdf). The expression for the $p d f$ is given below.

$$
\left.p d f_{p}\left(x_{i}, y_{i}\right)=\frac{1}{2 \pi \sigma_{i}(x) \sigma_{i}(y) \sqrt{1-\rho_{i}^{2}}} \times e^{[} \frac{-1}{2\left(\sqrt{1-\rho_{i}^{2}}\right)}[\text { aux }]\right]
$$

here,

$$
\operatorname{aux}=\left[\frac{x_{i}-\mu_{i}^{(x)}}{\sigma_{i}(x)}\right]^{2}+\left[\frac{y_{i}-\mu_{i}^{(y)}}{\sigma_{i}(y)}\right]^{2}-\frac{2 \pi\left[x_{i}-\mu_{i}^{(x)}\right]\left[y_{i}-\mu_{i}^{(y)}\right]}{\sigma_{i}(x) \sigma_{i}(y)}
$$

It is observed that the bidding co-efficients cannot be selected directly. (i) Here, first objective is to maximize the pdf by selecting the bidding co-efficients. (ii) The second objective is to maximize the profit of the suppliers and consumers. The above two observations are motivated to introduce two stage bacterial foraging algorithm. The description of the BFO algorithm and development of the proposed approach are given in the proceeding sections.

\section{BACTERIAL FORAGING OPTIMIZATION (BFO) ALGORITHM}

BFO [23, 24] algorithm is one of the biologically inspired optimization algorithms. In this section, the details such as background of bacteria and its foraging technique, short description of development of BFO algorithm and implementation of the said algorithm are provided.

\subsection{Background of bacteria and its foraging technique}

Bacteria chemical factories capable of bringing about significant changes in nature. BFO algorithm is based the foraging techniques of the E. coli bacterium cells present in the intestine of the human digestive system $[25,26]$. In the race of foraging for food location and survival, these cells with poor foraging techniques will be eliminated and those with good foraging techniques obtain enough food and reproduce their genes. As this process gradually intensifies the bacterium cells with poor foraging techniques are either extinct or redesigned. These foraging techniques and evolutionary principles helped scientists to hypothesize and correlate them with the optimization processes. A foraging animal always strives to ingest and gain energy by overcoming the physiological and environmental defects like poor sensing, cognitive capabilities and high density of prey, risky predators respectively.

\subsection{Development of BFO algorithm}

Typically, the BFOA consists of four main mechanisms: chemotaxis, swarming, reproduction and elimination-dispersal event.

\subsubsection{Chemotaxis}

In this process the movement of bacteria is enabled by helix-shaped flagellum. These set of rigid flagella helps the bacteria to swim. These flagella help the bacterium cells to move either by pushing them while rotating in an anti-clockwise direction by pulling them while rotating in the clockwise direction. This mechanism which enables the flagellum to spin by creating rotational forces is called bacterium motor. The biological motor can make the bacterium cells to swim or tumble. The E. coli bacterium cells maintain a specified direction during swimming and a random direction during tumbling. These alternate modes of operation are carried throughout its lifetime which enables them to search for nutrients.

$$
\theta^{i}(j+1, k, l)=\theta^{i}(j, k, l)+C(i) \times \frac{\Delta(i)}{\sqrt{\Delta^{T}(i) \Delta(i)}}
$$




\subsubsection{Swarming}

In this process, the bacterium cells swarm together just laid a group of swarming birds flying towards a certain direction during the migration season. When the bacterium cells reach a suitable location they start attacking other cells and converge at that particular location. This swarming pattern forms based on the dominance of the two stimuli relative distance between the respective bacterium from the signalling bacterium the performance of swarming from the signalling bacterium. The performance of swarming is to create a unique high dense bacterium for better foraging. The concentric patterns are a result of merged bacterium cells at the fittest point.

$$
\begin{gathered}
J_{c c}\left(\theta^{i}(j, k, l), P(j, k, l)\right)=\sum_{i=1}^{s} J_{c c}^{i}\left(\theta^{i}(j, k, l), P(j, k, l)\right) \\
J_{c c}\left(\theta^{i}(j, k, l), P(j, k, l)\right)=\sum_{i=1}^{s}\left[-d_{\text {attract }} \times \exp ^{\left.-w_{\text {attract }}\right]} \sum_{m=1}^{p}\left[\theta^{m}-\theta_{i}^{m}\right]^{2}\right. \\
+\sum_{i=1}^{s}\left[-h_{\text {repellent }} \times \exp ^{\left.-w_{\text {repellent }}\right]} \sum_{m=1}^{p}\left[\theta^{m}-\theta_{i}^{m}\right]^{2}\right.
\end{gathered}
$$

\subsubsection{Reproduction}

It is applied after chemotaxis, the process is provided below: (i) Fitness values of bacteria are store in ascending order, (ii) Bacteria with least fitness value are eliminated, (iii) The remaining are divided into two identical and filled at the same location.

\subsubsection{Elimination and dispersal}

The Process of swarming is affected when a group of bacteria gradually or suddenly reaches a new location due to the consumption of nutrients or by any other influence like attracting to fittest bacterium. This results in elimination of set of bacteria or dispersion from the respective group. The flowchart of the BFOA is provided in Figure 3.

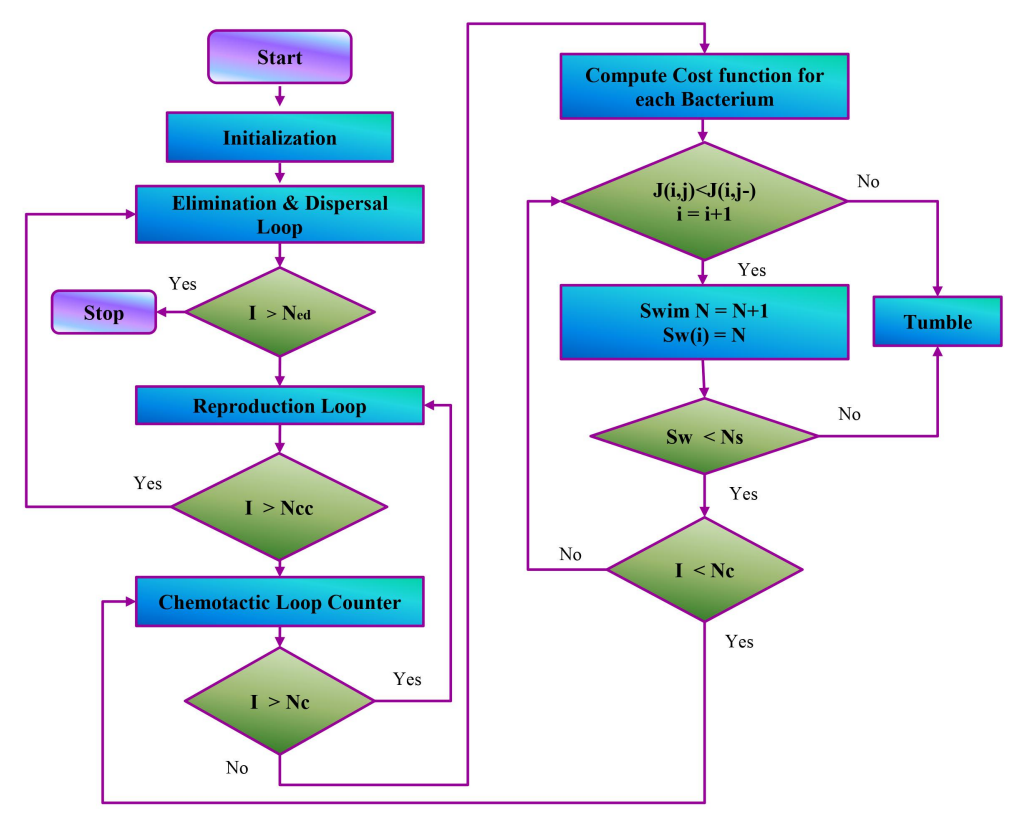

Figure 3. Flowchart of the BFOA 


\section{DEVELOPMENT OF THE BFO ALGORITHM}

The problem of supplier side optimal bidding strategy (DSOBS) is formulated in section 3 in this article. The objective is maximization of the profit of suppliers by selecting the bidding coefficients strategically. It is found from the survey of literature that the profit cannot be maximized by choosing all bidding coefficients independently. One of the bidding coefficients independently can be fixed. Now, the other bidding coefficient is determined by using any optimization method. This article presents the development of BFO algorithm for maximizing the profit of suppliers and consumers by choosing the appropriate bidding coefficients.

Step 1: $a$ is kept constant and $b$ is initialized. Range of the value of ' $b$ ' is [b 10b]

Step 2: The objective function is maximization of 'profit'. Again, BFO algorithm is used with control parameters shown in Table 1.

Step 3: Calculate

[1] MCP using (6)

[2] Power using (7)

[3] Profit of supplier using (2)

Step 4: Check equality constraint using eqn. (5), if 'yes' go to 'end'else.

Step 5: Apply operators of BFOA

Chemotaxis [Refer equation 12]

Swim [Refer equation 17]

Reproduction [Refer section 3.2.3]

Elimination [Refer section 3.2.4]

Step 6: Update the values of ' $b$ ' go to step 3. Repeat the steps from step 3 to 5 until either the maximum iterations are reached or equality constraint is met.

Step 7: Final result.

Table 1. Control parameters

\begin{tabular}{lll}
\hline S No & Control parameter & Value \\
\hline 1 & Population size (S) & 20 \\
2 & No. of Chemotaxis steps (Nc) & 30 \\
3 & Ns & 4 \\
4 & No. of repro steps (Nre) & 10 \\
5 & No. of elim-disp (Ned) & 5 \\
6 & C (i) & 0.05 \\
7 & Probability of elimi-disp (Ped) & 0.02 \\
\hline
\end{tabular}

\section{CASE STUDY}

The of the proposed algorithm has been developed in MATLAB (Version 2019). Applicability and simulation results of the proposed algorithm in comparison with existing algorithms has been done. Effectiveness of the proposed BFOA is tested on a standard system. Data of the system is available in [13] and presented in Table 2. The code has been executed on Personal laptop (8 GB RAM). The control parameters and their numerical values are shown in Table 1.

Table 2. Generator data

\begin{tabular}{lllll}
\hline Supplier & $\mathrm{e}$ & $\mathrm{f}$ & $P_{\min }$ & $P_{\max }$ \\
\hline 1 & 2.00 & 0.00375 & 20 & 160 \\
2 & 1.75 & 0.0175 & 15 & 150 \\
3 & 1.00 & 0.0625 & 10 & 120 \\
4 & 3.25 & 0.00834 & 10 & 100 \\
5 & 3.00 & 0.025 & 10 & 130 \\
6 & 3.00 & 0.025 & 10 & 130 \\
\hline
\end{tabular}

\subsection{Case 01}

BFO algorithm is tested on the IEEE 30 bus system. In this case, the values of $Q_{o}$ is $500 \mathrm{MW}$ and $\mathrm{K}$ is 0 . During the execution of the algorithm, the code is run for 20 times. Bidding coefficients, output powers and profits of the case study are presented in Table 3. 
Table 3. Simulations results of IEEE 30 bus system for case 01

\begin{tabular}{lllll}
\hline Supplier & $\mathrm{a}$ & $\mathrm{b}$ & Powers & Profit \\
\hline 1 & 2.00 & 0.038452 & 160 & 1208.351 \\
2 & 1.75 & 0.144258 & 58.24431 & 430.013 \\
3 & 1.00 & 0.211042 & 43.36678 & 279.3588 \\
4 & 3.25 & 0.082114 & 84.05669 & 521.2492 \\
5 & 3.00 & 0.12672 & 56.44106 & 324.0376 \\
6 & 3.00 & 0.155882 & 45.88207 & 275.5284 \\
& & & MCP & 10.15219 \\
& & & Profit & 3038.538 \\
\hline
\end{tabular}

It is observed from Table 3 that

- All output powers are within generator and load limits

- The proposed algorithm provides the best result in terms of profit within 20 seconds. Here the computational time is more because the algorithm has been tested for 1000 generations

\subsection{Case 02}

$\mathrm{BFO}$ algorithm is tested on the IEEE 30 bus system. In this case, the values of $Q_{o}$ is $300 \mathrm{MW}$ and K is 5. During the execution of the algorithm, the code is run for 20 times. Bidding coefficients, output powers and profits of the case study are presented in Table 4. It is observed from Table 4 that.

- All output powers are within generator and load limits

- The proposed algorithm provides the best result in terms of profit within 20 seconds. Here the computational time is more because the algorithm has been tested for 100 generations.

Error at each iteration for $300 \mathrm{MW}$ and $\mathrm{K}$ value of 5 is shown in Figure 4.

Table 4. Simulations results of IEEE 30 bus system for case 02

\begin{tabular}{lllll}
\hline Supplier & $\mathrm{a}$ & $\mathrm{b}$ & Powers & Profit \\
\hline 1 & 2.00 & 0.035525267 & 128.5317854 & 524.940744 \\
2 & 1.75 & 0.132519844 & 36.3426773 & 151.9170823 \\
3 & 1.00 & 0.208752673 & 26.66373495 & 103.979034 \\
4 & 3.25 & 0.081484179 & 40.69656181 & 121.1421314 \\
5 & 3.00 & 0.175007819 & 20.37695205 & 62.28627279 \\
6 & 3.00 & 0.24496562 & 14.55765887 & 46.61630903 \\
& & & MCP & 6.566125934 \\
& & & Profit & 1010.881574 \\
\hline
\end{tabular}

25

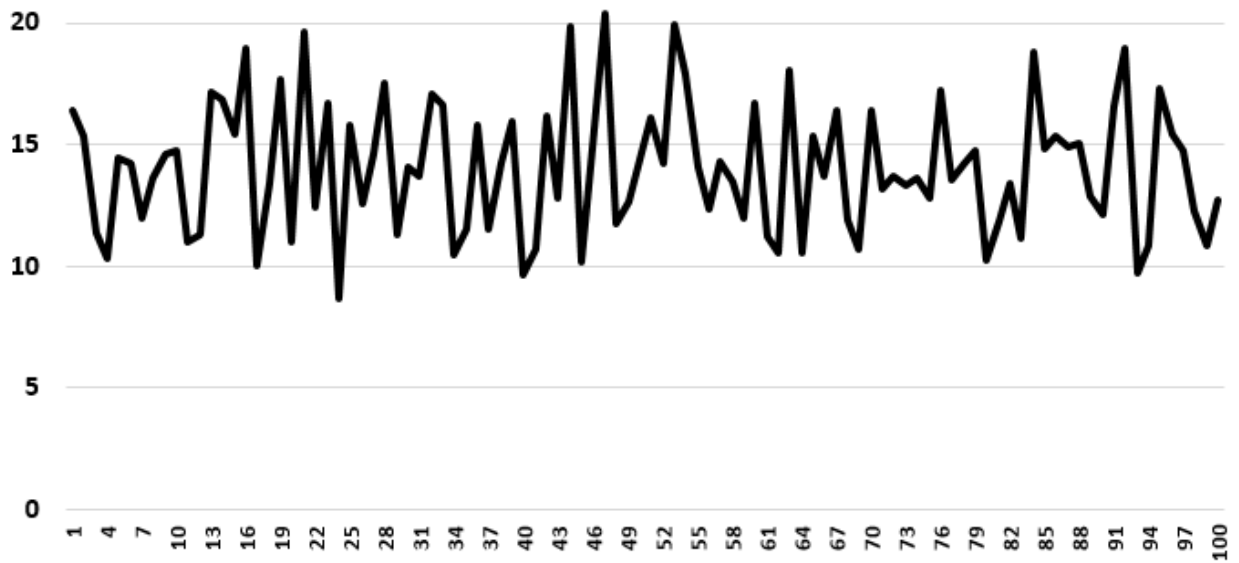

Figure 4. Error at each iteration for $300 \mathrm{MW}$ and $\mathrm{K}$ value of 5 


\subsection{Comparison and discussions}

The results of bidding coefficients of all methods are tabulated in Table 5. Profits of the proposed algorithm are compared with the existing methods and provided in Table 5. Comparison of MCP of all methods is given in Figure 5.

It has been found from these tables that the BFOA yields the best solution. Also, it is observed that the computational time is less. The same algorithm can be tested on large scale system in the real-time operation of electricity markets. It is identified that the variation of MCP and overall profit is due to

- The MCP is depending on bidding coefficients. For any small variation in bidding coefficients, it has more impact on the MCP

- The profit also is changed due to variation in $\mathrm{MCP}$

\subsection{Future scope}

In this article, the proposed algorithm has applied for single side optimal bidding strategy problem to maximize the profit of the players. The algorithm can be applied for SSOBS by considering security constraints on transmission lines. Also, the proposed algorithm may be applied for the same problem with consideration of reserve constraints.

Table 5. Profits of suppliers

\begin{tabular}{lllll}
\hline Generator & GA[13] & PSO[13] & FAGSA [13] & Proposed \\
\hline 1 & 741.45 & 772.41 & 1034.9 & 1208.351 \\
2 & 321.32 & 340.10 & 376.38 & 430.013 \\
3 & 119.33 & 125.06 & 157.22 & 279.3588 \\
4 & 261.01 & 280.36 & 498.47 & 521.2492 \\
5 & 125.56 & 136.32 & 275.38 & 324.0376 \\
6 & 125.56 & 136.22 & 275.38 & 275.5284 \\
Total Profit & 1694.23 & 1790.57 & 2617.73 & 3038.538 \\
\hline
\end{tabular}

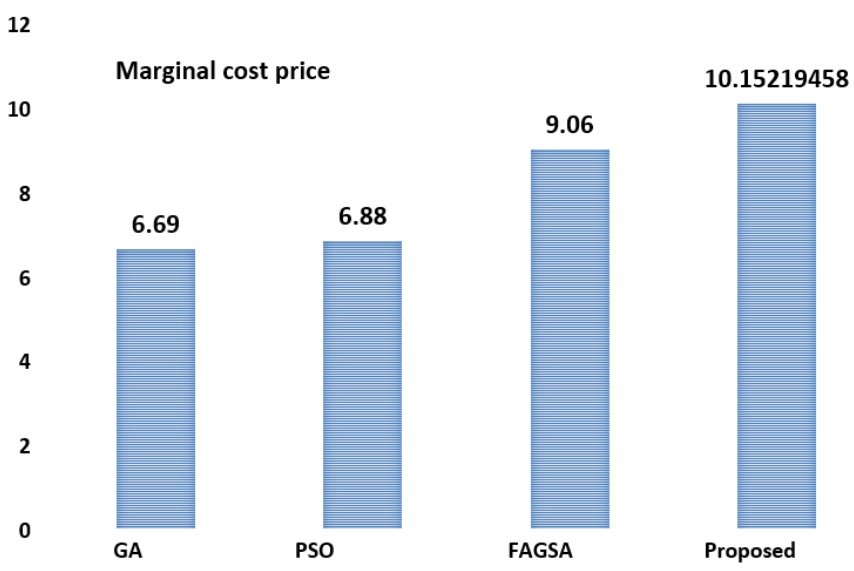

Figure 5. Comparision of MCP of all methods

\section{CONCLUSIONS}

Single side optimal bidding strategy (SSOBS) has been successfully solved using the BFO algorithm. Simulation results in terms of bidding co-efficients, output powers and profits for various loads are provided. Also, the results of the proposed algorithm are compared with the available methods. It is observed that the proposed BFO algorithm gives better results. It can noticed in the simulation results that The MCP is depending on bidding coefficients. For any small variation in bidding coefficients, it has more impact on the MCP. Also, the profit is varied due to the variation in the MCP. The proposed algorithm can be used for various other problems in operation of power system due to its inherent capabilities in finding the global solution. Also the same algorithm can be implemented for real time electricity markets including smart grid with renewable energy sources. 


\section{CONFLICT OF INTEREST}

The authors declare that they have no conflict of interest.

\section{ACKNOWLEDGEMENT}

Authors are thankful to the reviewers for their useful comments to improvize the manuscript. We are grateful to JNT University, Kakinada, BITS Pilani, KK Birla Goa Campus and Chalapathi Institute of Technology, Guntur for providing the infrastructure to carryout the present work.

\section{REFERENCES}

[1] 'Link Lowy Institute Asia Power Index 2019," Published by Lowy Institute, 2019.

[2] Kwok. W., "Restructured Electric Power Systems and Electricity Markets," Restructured Electric Power Systems Analysis of Electricity Markets with Equilibrium Models, Hoboken, New Jersey: John Wiley sons, inc., publication, 2010.

[3] F. Wen and A. K. David, "Optimal bidding strategies for competitive generators and large consumers," Elect. Power Syst. Res., vol. 23, pp. 7-43, Apr 2001.

[4] A. K. David, "Competitive bidding in electricity supply," IEE Proceedings C - Generation, Transmission and Distribution, vol. 140, no. 5, pp. 421-426, Sept. 1993.

[5] Jain, A., Srivastava, S.C., "Strategic Bidding and Risk Assessment Using Genetic Algorithm in Electricity Markets," International Journal of Emerging Electric Power Systems, vol. 10, no. 5, 2009.

[6] Ferrero, R. W., Ramesh, V. C., and Shahidehpour, S. M., "Transaction analysis in deregulated power system using game theory," IEEE Trans Power Syst., vol. 12, no. 3, pp. 1340-1347, 1997.

[7] S. de la Torre, J. Contreras and A. J. Conejo, "Finding multiperiod Nash equilibria in pool-based electricity markets," IEEE Transactions on Power Systems, vol. 19, no. 1, pp. 643-651, Feb. 2004.

[8] Gross, G., and Finlay, D., "Generation supply bidding in perfectly competitive electricity markets," Comput. Math. Organ. Theory, vol. 6, pp. 83-98, 2000.

[9] H. Song, C. -. Liu, J. Lawarree and R. W. Dahlgren, "Optimal electricity supply bidding by Markov decision process," IEEE Transactions on Power Systems, vol. 15, no. 2, pp. 618-624, May 2000.

[10] Hobbs, B. F., and Helman, U., "Complementary-based equilibrium modeling for electric power markets" Bunn, D. (Ed.): 'Modeling prices in competitive electricity markets' Wiley, New York, 2004.

[11] C. W. Richter, G. B. Sheble and D. Ashlock, "Comprehensive bidding strategies with genetic programming/finite state automata," IEEE Transactions on Power Systems, vol. 14, no. 4, pp. 1207-1212, 1999.

[12] David, A.K., and Wen, F., "Strategic bidding for electricity supply in a day-ahead energy market," Electr. Power Syst. Res., vol. 59, pp. 197-206, 2001.

[13] J. Vijaya Kumar, D.M. Vinod Kumar, "Generation bidding strategy in a pool based electricity market using Shuffled Frog Leaping Algorithm," Applied Soft Computing, vol. 21, pp. 407-414, 2014.

[14] Ahmet D.Yucekaya, JorgeValenzuela, GerryDozier, "Strategic bidding in electricity markets using particle swarm optimization," Electric Power Systems Research, vol. 79, no. 2, pp. 335-345, Feb, 2009.

[15] V. V. S. Angatha, K. Chandram, A. J. Laxmi, ”Bidding Strategy in Deregulated Power Market Using Differential Evolution Algorithm", Journal of Power and Energy Engineering, vol. 3, pp. 37-46, 2015.

[16] Sudhakar A. V. V., Karri, C., Laxmi A. J., "Optimal Bidding Strategy in Deregulated Power Market Using Invasive Weed Optimization," Applications of Artificial Intelligence Techniques in Engineering. Springer, pp. 421-429, 2019.

[17] C. Karri, D. Rajababu and K. Raghuram., "Optimal Bidding Strategy in Deregulated Power Market Using Krill Herd Algorithm,", "Applications of Artificial Intelligence Techniques in Engineering. Springer, pp. 43-51, 2019.

[18] J. Vijaya Kumar and D. M. Vinod Kumar, " Optimal Bidding Strategy in a Pool type Electricity Market using Bacterial Foraging Algorithm," Journal of Electrical Systems, vol. 8, no. 3, pp. 328-337, 2012

[19] S. M. Ali Naseri Javareshk, S. A. Kouche Biyouki and S. H. Darban, "Optimal Bidding Strategy in Double-Sided Auctions of the Competitive Power Market Using Agent-Based Algorithm," 27th Iranian Conference on Electrical Engineering (ICEE), pp. 798-803, 2019.

[20] T. Niknam, S. Sharifinia, R. Azizipanah-Abarghooee, "A new enhanced bat-inspired algorithm for finding linear supply function equilibrium of GENCOs in the competitive electricity market," Energy Convers. Manage., vol. 76, pp. 1015-1028, 2013. 
[21] B. P. Majumder, M. N. Faqiry, S. Das and A. Pahwa, "An efficient iterative double auction for energy trading in microgrids," IEEE Symposium on Computational Intelligence Applications in Smart Grid (CIASG), pp. 1-7, 2014.

[22] S. Singh and M. Fozdar, "Optimal bidding strategy with the inclusion of wind power supplier in an emerging power market," IET Generation, Transmission and Distribution, vol. 13, no. 10, pp. 1914-1922, 2019.

[23] Passino K.M, "Biomimicry for optimization, control and automation," Springer, pp. 768-818, 2015.

[24] Berg, H., "Random Walks in Biology," Princeton Univ. Press, Princeton, 1993.

[25] Das S., Biswas A., Dasgupta S., Abraham A., "Bacterial Foraging Optimization Algorithm: Theoretical Foundations, Analysis, and Applications," Foundations of computational intelligence. Springer, vol. 3, pp. 23-55, 2009.

[26] S. Mishra and C. N. Bhende, "Bacterial Foraging Technique-Based Optimized Active Power Filter for Load Compensation,” IEEE Transactions on Power Delivery, vol. 22, no. 1, pp. 457-465, Jan. 2007

\section{BIOGRAPHIES OF AUTHORS}

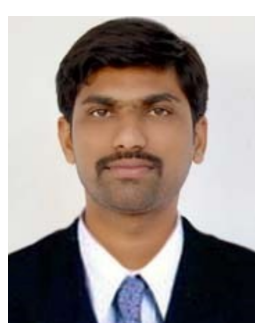

Uday Kiran $\mathbf{L}$ received the B. Tech. and M. Tech. degrees in Electrical Engineering from the Jawaharlal Nehru Technological University, Hyderabad, Telangana, India, in 2007 and 2010. He is currently working as Associate Professor at Chalapathi Institute of Technology, Guntur, Andhra Pradesh. Pursuing his Ph.D in Deregulated Power Systems at Jawaharlal Nehru Technological University, Kakinada, Andhra Pradesh, India. His research interests include power systems, restructuring of power systems, deregulated power systems, and power market, power economics, energy analysis and power demand forecasting.

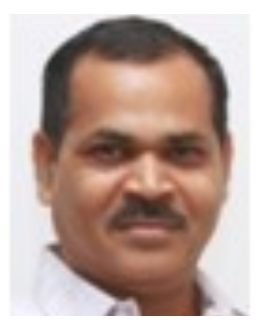

Sivanagaraju S received his Masters degree in 2000 from IIT, Kharagpur and did his Ph.D from Jawaharlal Nehru Technological University, Hyderabad in 2004.He is currently working as Professor in the Department of Electrical and Electronics Engineering, Jawaharlal Nehru Technological University Kakinada, Andhara Pradesh, India. His interests include FACTS controllers, electrical distribution system automation, optimization techniques, voltage stability, power system analysis, and power system operation and control

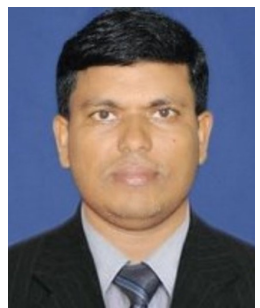

Chandram Karri has received the B. Tech in Electrical and Electronics Engineering in 2000. He received Ph.D. degree in Electrical engineering from NIT Warangal in 2010. In 2009, he joined as an Assistant Professor in Department of Electrical Engineering in BITS Pilani Goa Campus. The Current area of research including FACTS controllers and their applications in power distribution systems, biologically inspired algorithms and their applications in power system operation in deregulation, power quality detection and mitigation, signal processing, Multi objective algorithms applications in power systems and power system operation and control. He has published 25 international journal articles, 21 international conference articles and 2 book chapters. 